\title{
MARKET RISK AND MONETARY DYNAMICS
}

Xiaolou Yang, Department of Accounting and Finance, Youngstown State University, OH, U.S.A.

\author{
dx.doi.org/10.18374/JIFE-20-3.5
}

\begin{abstract}
Monetary policy making has to incorporate various sources of uncertainties. Ignorance of this consideration in the monetary model causes a gap between the promise of theory and the reality of practice. This study investigates the various effects of economic risks and uncertainties on monetary policy making. In particularly, I show that without accounting for the economics uncertainties, the traditional theoretical model could generate a serious distorted macroeconomic outcomes, and consequently resulting inefficient policy and rules. On the other hand, when these uncertainties are taken into account, the model shows a cautionary effect and are less sensitive to the shocks of the economy. Moreover, this study illustrate the effect of the cautionary effect associated with the uncertainty and, when future uncertainties increases, the trade-off between "caution" and "intensity".
\end{abstract}

Keywords: risk, monetary dinamics, 